\title{
Maladies chroniques et taux de mortalité au sein des populations autochtones du Canada : tirer profit des connaissances
}

\author{
M. King, Ph. D., directeur scientifique, Instituts de recherche en santé du Canada, Institut de la santé des Autochtones
}

*Cet article fait l'objet d'une publication conjointe par Preventing Chronic Disease et Maladies chroniques au Canada. Maladies chroniques au Canada en est le premier éditeur, Preventing Chronic Disease le second.

Il est malheureux de constater que les populations autochtones au Canada ont une espérance de vie considérablement plus faible que celle des populations non autochtones, et ce, qu'elles vivent dans des communautés rurales ou en milieu urbain ${ }^{1}$. Les disparités entre l'état de santé des Autochtones du Canada et celui des autres Canadiens sont un sujet constant de préoccupation ${ }^{2}$; la compréhension de ces disparités passe par l'identification et la compréhension des déterminants sociaux de la santé, éléments clés, selon moi, pour la résolution du problème. Il faut être cependant conscients qu'il existe pour les populations autochtones des déterminants sociaux spécifiques, associés à leur culture, à leur histoire et à la colonisation, ainsi qu'au contexte social, économique, politique et géographique actuel ${ }^{3}$.

Bruce et coll. ${ }^{4}$ ont analysé la question de l'obésité et des affections liées à l'obésité (dyslipidémie, hypertension et diabète) dans une communauté des Premières nations du Manitoba. Ils ont constaté que, comme au sein d'autres populations autochtones (p. ex. en Saskatchewan, la province voisine ${ }^{5}$ ), la prévalence de l'obésité et des affections liées à l'obésité dans cette population est plus élevée chez les femmes que chez les hommes, et que les affections liées à l'obésité sont courantes même chez les jeunes adultes. L'hypertension non diagnostiquée est également très fréquente. De leur côté, Riediger et coll. ${ }^{6}$ ont observé, toujours dans cette communauté, un risque de maladies cardiovasculaires élevé en raison des faibles taux plasmatiques d'apolipoprotéine A1, en particulier chez les femmes.

$\mathrm{Ng}$ et coll. ${ }^{7}$ ont étudié la prévalence de l'arthrite dans les populations autochtones au Canada, comparant les disparités entre les populations des territoires du Nord et celles des dix provinces (" le Sud »). Ils ont utilisé les données de l'Enquête auprès des peuples autochtones (EAPA) de 2006, une enquête post-censitaire menée par Statistique Canada incluant des questions sur la santé et sur les aspects économiques et sociaux. L'EAPA visait les Inuits, les Métis et les membres des Premières nations vivant hors réserve; les membres des Premières nations vivant dans les réserves ont été suivis pour leur part grâce à l'Enquête régionale longitudinale sur la santé des Premières nations de 2002-2003 menée par l'Assemblée des Premières Nations ${ }^{8}$. Grâce au suréchantillonnage des résidants des territoires du Nord dans l'EAPA, il a été possible de faire des comparaisons efficaces avec le Sud, davantage peuplés. L'arthrite et les rhumatismes constituent le problème de santé le plus couramment signalé par les Autochtones du Canada. Dans le Sud, où réside plus de $90 \%$ de la population autochtone, la prévalence globale était de $20,1 \%$, et de $25,3 \%$ chez les membres des Premières nations vivant hors réserve. La prévalence globale de l'arthrite et des rhumatismes dans les territoires du Nord était considérablement plus faible $(12,7 \%$ pour l'ensemble de la population), et plus faible également chez les membres des Premières nations et des Inuits du Nord que chez ceux du Sud. Les sujets souffrant d'arthrite indiquent en plus forte proportion être atteints d'au moins une autre maladie chronique, ils sont plus nombreux à consulter des professionnels de la santé et moins nombreux à occuper un emploi ce qui n'est sans doute guère surprenant -, sans cependant qu'une relation de cause à effet ait pu être établie.

Tjepkema et coll. ${ }^{1}$ ont étudié les taux de mortalité chez les adultes autochtones vivant en milieu urbain sur une période de 11 ans (1991 à 2001), en mettant en relation les données du registre des décès, celles des recensements et celles des déclarations de revenus. Ils ont ainsi obtenu une cohorte d'adultes autochtones vivant en milieu urbain composée de près de 25500 sujets, sur une cohorte totale de 2,6 millions de résidants en milieu urbain. La variable principale, l'espérance de vie résiduelle à 25 ans, est considérablement plus faible pour les Autochtones vivant en milieu urbain que pour les Canadiens non autochtones vivant en milieu urbain. Elle est plus courte de 4,7 ans chez les hommes et de 6,5 ans chez les femmes autochtones. Ces résultats sont similaires à ceux publiés récemment par les mêmes chercheurs à propos des résidants des réserves et des résidants des zones non urbaines ${ }^{9}$; ils font également ressortir le même biais lié au sexe, au sens où l'espérance de vie des femmes autochtones dépasse celle des hommes autochtones d'environ deux ans? Les chercheurs ont constaté que les deux principales causes de mortalité chez les Autochtones du Canada sont les maladies de l'appareil circulatoire et le cancer. Par rapport à la population non autochtone, les taux de maladies de l'appareil circulatoire sont élevés aussi bien chez les hommes que chez les femmes, les taux de cancer étant élevés uniquement chez les femmes autochtones ${ }^{1}$. Des causes spécifiques de décès sont également associées à des taux relativement élevés, en particulier les causes liées à l'alcool et les causes externes, dont les suicides et les accidents. Par ailleurs, les taux de décès susceptibles d'être évités par intervention médicale sont élevés aussi bien chez les hommes que chez les femmes ${ }^{1}$.

Il est important de pouvoir décrire et comprendre les disparités liées à l'état de santé et les facteurs de risque associés aux maladies chroniques affligeant les populations autochtones du Canada, mais nous devons comprendre l'ensemble des causes avant de mettre en œuvre des politiques permettant de lutter contre ces problèmes, de 
prévenir leur apparition et d'inverser leur tendance à l'augmentation. Les champs d'intérêt des quatre études présentées ici vont de la simple description à la compréhension approfondie des facteurs de risque d'ordres physique, socioéconomique et social. La prochaine étape, probablement l'étape cruciale, consistera à utiliser ces connaissances pour corriger les disparités. L'étude menée par Bruce et coll. ${ }^{4}$ est, à cet égard, porteuse d'espoir : la communauté est engagée dans des projets visant à résoudre et à prévenir les problèmes liés à l'obésité, ce qui permettra peut-être même d'inverser ce que les facteurs de risque laissent apparaître comme une tendance à l'augmentation. Nous avons appris, sans en connaître tous les détails, qu'un projet de prévention du diabète gestationnel par le contrôle de la prise de poids pendant la grossesse est en cours; que la communauté gère un centre de conditionnement physique; que le centre de santé a lancé une initiative d'éducation en matière d'alimentation, d'activité physique et de mieuxêtre; que des groupes de marche sont organisés et que des programmes d'activité physique sont offerts dans les écoles. Dans tous ces projets, les chercheurs travaillent de concert avec la communauté. Ce niveau de suivi n'est pas envisageable dans le cas des données d'enquêtes nationales, étant donné que l'engagement de la communauté locale est en général nécessaire pour utiliser les données sur la santé et transformer les comportements et les environnements associés. Il demeure que la collaboration avec les organisations nationales est essentielle pour que les connaissances liées à la santé conduisent à des politiques et des mesures concrètes.

La nécessité de réduire les inégalités en matière de santé chez les Autochtones figure parmi les principales orientations stratégiques des Instituts de recherche en santé du Canada (IRSC) ${ }^{10}$. L'Institut de la santé des Autochtones des IRSC soutient et va continuer à soutenir - l'engagement communautaire, le renforcement des capacités et le développement du réseau, outils clés pour assurer l'équité en matière de santé pour les Autochtones. Nous devons cependant aller au-delà de ces engagements, et appuyer la rechercheaction (qu'est-ce qui fonctionne, qu'est-ce qui ne fonctionne pas et pourquoi) et les transferts de connaissances (adaptation aux différents contextes et changements d'échelle) en vue de définir des modèles de bonnes pratiques permettant à nos communautés d'atteindre leurs objectifs d'équité en matière de santé. Cet objectif étant irréalisable sans une prise en compte globale, incluant les dimensions mentale, physique, émotionnelle et spirituelle de nos vies, nous devons voir plus loin que les déterminants classiques de la santé et tenir compte de facteurs comme la promotion de la résilience par le biais de la spiritualité, de la culture, de la revitalisation linguistique, des activités traditionnelles et toutes les autres formes d'épanouissement culturel $^{3}$. Grâce au suivi effectué par les chercheurs et à l'engagement de la communauté aux échelles locale, régionale et nationale, nous espérons éliminer à terme, au Canada, les disparités entre l'état de santé des Autochtones et celui de l'ensemble de la population non autochtone.

\section{Références}

1. Tjepkema $M$, Wilkins $R$, Senécal $S$, Guimond E, Penney C. La mortalité chez les adultes autochtones vivant en milieu urbain au Canada, 1991-2001. Maladies chroniques au Canada 2010;31(1):5-25.

2. Lix, L.M, S. Bruce, J. Sarkar, T.K. Young. Facteurs de risque et problèmes de santé chroniques chez les Autochtones et les non-Autochtones. Rapports sur la santé 2009;20(4):23-32.

3. King $M$, Smith A, Gracey M. Indigenous health part 2: the underlying causes of the health gap. Lancet 2009;374:76-85.

4. Bruce SG, Riediger ND, Zacharias JM, Young TK. Obesity and obesityrelated comorbidities in a Canadian First Nation population. Prev Chronic Dis 2011;8(1). Consultable en ligne à la page : http://www.cdc.gov/pcd/issues/2011/ jan/09_0212.htm

5. Dyck R, Osgood N, Lin TH, Gao A, Stang MR. Epidemiology of diabetes mellitus among First Nations and non-First Nations adults. CMAJ 2010;182:244-56.
6. Riediger ND, Bruce SG, Young TK. Cardiovascular risk according to plasma apolipoprotein and lipid profiles in a Canadian First Nation. Prev Chronic Dis 2011;8(1). Consultable en ligne à la page : http://www.cdc.gov/pcd/issues/2011/ jan/09_0216.htm

7. Ng C, Chatwood S, Young TK. Arthrite dans la population autochtone canadienne : différences entre le Nord et le Sud en ce qui a trait à la prévalence et aux corrélats. Maladies chroniques au Canada 2010;31(1):26-31 .

8. Enquête régionale longitudinale sur la santé des Premières Nations (ERS) [Internet]. Ottawa (Ont.), Assemblée des Premières nations, 2005 [cité le 7 juin 2010]. Consultable en ligne à la page : http://rhs-ers.ca/francais/index.asp

9. Tjepkema M., R. Wilkins, S. Senécal, E. Guimond, C. Penney. La mortalité chez les Métis et les Indiens inscrits adultes au Canada : étude de suivi sur 11 ans. Rapports sur la santé 2009;20(4):33-55.

10. L'innovation au service de la santé : de meilleurs soins et services par la recherche [Internet]. Ottawa (Ont.), Instituts de recherche en santé du Canada, oct. 2009 [cité le 14 juillet 2010]. http://www.cihr. $\mathrm{ca} / \mathrm{f} / 40490 . \mathrm{html}$ 